Related content

- Particle Dark Matter constraints: the effect
of Galactic uncertainties
Maria Benito, Nicolás Bernal, Nassim
Bozorgnia et al.
- Erratum: Measuring global monopole
$\frac{\text { velocities, one by one }}{\text { Asier Lopez-Eiguren, Jon Urrestilla and }}$
Ana Achúcarro
- Erratum: primordial spikes from wrapped
brane inflation
Takeshi Kobayashi and Jun'ichi Yokoyama

View the article online for updates and enhancements.

\section{Erratum: Particle Dark Matter constraints: the effect of Galactic uncertainties}

To cite this article: Maria Benito et al JCAP06(2018)E01

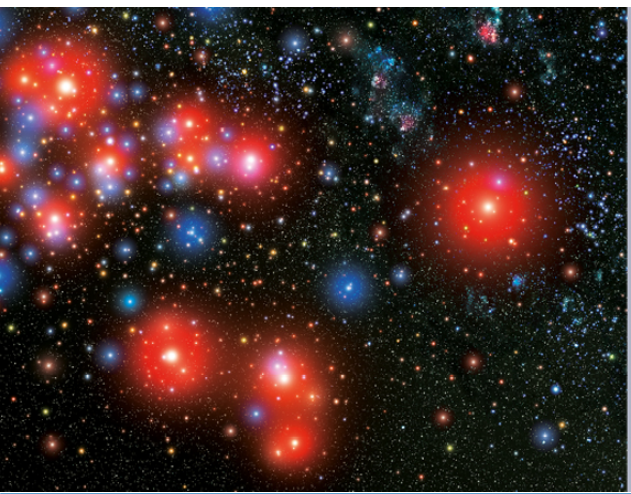

$\frac{\text { A.A }}{\mathbf{S}}$ IOP Astronomy ebooks

Part of your publishing universe and your first choice for astronomy, astrophysics, solar physics and planetary science ebooks. iopscience.org/books/aas 


\title{
Erratum: Particle Dark Matter constraints: the effect of Galactic uncertainties
}

\author{
Maria Benito, ${ }^{a}$ Nicolás Bernal, ${ }^{a, b}$ Nassim Bozorgnia, ${ }^{c}$ \\ Francesca Calore $^{c, d}$ and Fabio locco ${ }^{a}$
}

\author{
${ }^{a}$ ICTP South American Institute for Fundamental Research, \\ Instituto de Física Teórica - Universidade Estadual Paulista (UNESP), \\ Rua Dr. Bento Teobaldo Ferraz 271, 01140-070 São Paulo, SP Brazil \\ ${ }^{b}$ Centro de Investigaciones, Universidad Antonio Nariño, \\ Cra 3 Este \# 47A-15, Bogotá, Colombia \\ ${ }^{c}$ GRAPPA Institute, Institute for Theoretical Physics Amsterdam \\ and Delta Institute for Theoretical Physics, University of Amsterdam, \\ Science Park 904, 1098 XH Amsterdam, The Netherlands \\ ${ }^{d}$ LAPTh, CNRS, 9 Chemin de Bellevue, 74941 Annecy-le-Vieux, France \\ E-mail: mariabenitocst@gmail.com,nicolas.bernal@uan.edu.co, \\ n.bozorgnia@uva.nl, calore@lapth.cnrs.fr, fabio.iocco.astro@gmail.com
}

Received June 4, 2018

Accepted June 4, 2018

Published June 11, 2018

Erratum to: JCAP02(2017)007

ArXiv ePrint: 1612.02010

\section{Erratum}

This erratum corrects the following mistakes in our paper "Particle Dark Matter Constraints: the Effect of Galactic Uncertainties": values of the stellar mass of the Milky Way (MW) in column 4 of table 1, values of the total MW mass quoted in text, and a typo when referring to the value of the total MW as quoted in the Sagittarius stream paper [1].

Here we provide a correct version of table 1 (stellar mass of the MW corrected, column 4), and replace the final, corrected version of the text (original lines below table 1 in page 5).

No other element of the paper has changed, and conclusions are unaffected. 


\begin{tabular}{|c|c|c|c|c|c|c|}
\hline Morphology & $R_{0}(\mathrm{kpc})$ & $v_{0}(\mathrm{~km} / \mathrm{s})$ & $M_{*}\left(\times 10^{10} M_{\odot}\right)$ & $\gamma$ & $\rho_{0}\left(\mathrm{GeV} / \mathrm{cm}^{3}\right)$ & Reference \\
\hline $\mathrm{BjX}$ & 8 & 230 & $4.6_{-0.5}^{+0.6}$ & $1.11_{-0.03}^{+0.04}$ & $0.466 \pm 0.010$ & {$[2-5]$} \\
\hline $\mathrm{BjX}$ & 7.5 & 312 & $4.2_{-0.5}^{+0.6}$ & $0.633_{-0.020}^{+0.019}$ & $1.762 \pm 0.017$ & {$[2-5]$} \\
\hline $\mathrm{BjX}$ & 8.5 & 180 & $5.1_{-0.6}^{+0.7}$ & $2.02 \pm 0.07$ & $0.055 \pm 0.004$ & {$[2-5]$} \\
\hline $\mathrm{FkX}$ & 8 & 230 & $4.3 \pm 0.5$ & $1.38_{-0.02}^{+0.03}$ & $0.427_{-0.008}^{+0.007}$ & {$[4-7]$} \\
\hline $\mathrm{DiX}$ & 8 & 230 & $5.6 \pm 0.7$ & $0.43_{-0.06}^{+0.07}$ & $0.405 \pm 0.011$ & {$[4,5,8,9]$} \\
\hline $\mathrm{CjX}$ & 8 & 230 & $4.8_{-0.6}^{+0.7}$ & $1.03 \pm 0.04$ & $0.471_{-0.011}^{+0.010}$ & {$[3-5,10]$} \\
\hline FiX & 8 & 230 & $5.2 \pm 0.6$ & $0.82 \pm 0.05$ & $0.387 \pm 0.010$ & {$[4-6,9]$} \\
\hline
\end{tabular}

Table 1. We adopt a gNFW density profile with $R_{s}=20 \mathrm{kpc}$. From left to right we report the nomenclature adopted for each morphology, the values of Galactic parameters $\left(R_{0}, v_{0}\right)$, the baryonic mass in the Galaxy for that specific baryonic morphology, the best-fit values of index $\gamma$ and $\rho_{0}$ according to the procedure described in the text, the value of $\chi^{2}$ per degree of freedom, and the references for the three-dimensional morphology shape. Quoted uncertainties are $1 \sigma$ (68\% confidence level) here and elsewhere unless otherwise stated. The criteria that led to the choice of these morphologies are explained in the text.

\subsection{Changes}

When varying Galactic parameters, we obtain the total mass of the MW within $50 \mathrm{kpc}$ to be in the range $\mathrm{M}(<50)=(1.2-22.9) \times 10^{11} \mathrm{M}_{\odot}$. The lower limit is in agreement with previous determinations $[1,11-14]$, while the larger MW masses we obtain can not be directly compared, as the adopted Galactic Parameters are different than ours. When varying baryonic morphology, the minimum/maximum values obtain are $\mathrm{M}(<50 \mathrm{kpc})=4.36_{-0.10}^{+0.11} \times 10^{11} \mathrm{M}_{\odot}$ and $\mathrm{M}(<50 \mathrm{kpc})=7.0 \pm 0.3 \times 10^{11} \mathrm{M}_{\odot}$. The former value for the variation of morphology is in good agreement with mass estimate from kinematics of globular clusters, satellite galaxies and halo stars $[11,12,14,15]$. There is, however, a discrepancy at the $1 \sigma$ level with the independent determination in [1], that used the Sagittarius stream, and is slightly smaller than the other cited determinations. All our results are in agreement at the $1 \sigma$ level with the recent estimate of the dynamical mass [16] within the region of the Galactic bulge, as in the analysis presented in [17]

\section{References}

[1] S.L.J. Gibbons, V. Belokurov and N.W. Evans, 'Skinny Milky Way please', says Sagittarius, Mon. Not. Roy. Astron. Soc. 445 (2014) 3788 [arXiv:1406.2243] [INSPIRE].

[2] E. Dwek et al., Morphology, near infrared luminosity and mass of the galactic bulge from Cobe dirbe observations, Astrophys. J. 445 (1995) 716 [INSPIRE].

[3] C. Han and A.P. Gould, Stellar contribution to the Galactic bulge microlensing optical depth, Astrophys. J. 592 (2003) 172 [astro-ph/0303309] [INSPIRE].

[4] K. Ferriere, W. Gillard and P. Jean, Spatial distribution of interstellar gas in the innermost 3 kpc of our Galaxy, Astron. Astrophys. 467 (2007) 611 [astro-ph/0702532] [INSPIRE].

[5] K. Ferriere, Global Model of the Interstellar Medium in Our Galaxy with New Constraints on the Hot Gas Component, Astrophys. J. 497 (1998) 759. 
[6] A.C. Robin, D.J. Marshall, M. Schultheis and C. Reyle, Stellar populations in the Milky Way bulge region: Towards solving the Galactic bulge and bar shapes using 2MASS data, Astron. Astrophys. 538 (2012) A106 [arXiv:1111.5744] [INSPIRE].

[7] S. Calchi Novati and L. Mancini, Microlensing towards the LMC: self lensing for OGLE-II and OGLE-III, Mon. Not. Roy. Astron. Soc. 416 (2011) 1292 [arXiv:1105.4615] [INSPIRE].

[8] N. Bissantz and O. Gerhard, Spiral arms, bar shape and bulge microlensing in the milky way, Mon. Not. Roy. Astron. Soc. 330 (2002) 591 [astro-ph/0110368] [INSPIRE].

[9] J. Bovy and H.-W. Rix, A Direct Dynamical Measurement of the Milky Way's Disk Surface Density Profile, Disk Scale Length and Dark Matter Profile at $4 k p c \lesssim R \lesssim 9 k p c$, Astrophys. J. 779 (2013) 115 [arXiv: 1309.0809] [INSPIRE].

[10] E. Vanhollebeke, M.A.T. Groenewegen and L. Girardi, Stellar populations in the Galactic bulge, arXiv:0903.0946 [INSPIRE].

[11] M.I. Wilkinson and N.W. Evans, The present and future mass of the Milky Way halo, Mon. Not. Roy. Astron. Soc. 310 (1999) 645 [astro-ph/9906197] [INSPIRE].

[12] T. Sakamoto, M. Chiba and T.C. Beers, The Mass of the Milky Way: Limits from a newly assembled set of halo objects, Astron. Astrophys. 397 (2003) 899 [astro-ph/0210508] [INSPIRE].

[13] M.C. Smith et al., The RAVE Survey: Constraining the Local Galactic Escape Speed, Mon. Not. Roy. Astron. Soc. 379 (2007) 755 [astro-ph/0611671] [INSPIRE].

[14] A.J. Deason, V. Belokurov, N.W. Evans and J.H. An, Broken Degeneracies: The Rotation Curve and Velocity Anisotropy of the Milky Way Halo, Mon. Not. Roy. Astron. Soc. 424 (2012) L44 [arXiv: 1204 .5189] [INSPIRE].

[15] G. Besla et al., Are the Magellanic Clouds on their First Passage about the Milky Way?, Astrophys. J. 668 (2007) 949 [astro-ph/0703196] [INSPIRE].

[16] M. Portail, C. Wegg, O. Gerhard and I. Martinez-Valpuesta, Made-to-measure models of the galactic box/peanut bulge: stellar and total mass in the bulge region, Mon. Not. Roy. Astron. Soc. 448 (2015) 713 [arXiv: 1502.00633].

[17] F. Iocco and M. Benito, An estimate of the DM profile in the Galactic bulge region, Phys. Dark Univ. 15 (2017) 90 [arXiv:1611.09861] [INSPIRE]. 